\title{
Correction to: spatial positioning of individuals in a group of feral horses: a case study using drone technology
}

\author{
Sota Inoue ${ }^{1} \cdot$ Shinya Yamamoto ${ }^{2} \cdot$ Monamie Ringhofer $^{2} \cdot$ Renata S. Mendonça $^{3} \cdot$ Carlos Pereira $^{4} \cdot$ Satoshi Hirata $^{1}$
}

Published online: 25 May 2019

(C) Mammal Research Institute, Polish Academy of Sciences, Białowieża, Poland 2019

\section{Correction to: Clinical Rheumatology https://doi.org/10.1007/s13364-018-0400-2}

The original version of this article, unfortunately contained errors.

In the Abstract section originally reading, "(1) the male in between social and spatial relationships indicated that they are independent from each other. The present study is the first to reveal the characteristics of spatial positioning in a mammalian group using drone technology." should be read as "(1) the male in". Under the section "Distribution of distance between individuals" the correct data should have been "... K-S test, $\mathrm{n}=1708$..." instead of “...-S test, $\mathrm{n}=1708 . .$. " and under the section "Social network analysis", the sentence "We found a significant correlation between the grooming and inter-individual distance networks
(QAP test, $\mathrm{r}=0.51, \mathrm{p}<0.01$ ) but not between the grooming and proximity networks (QAP test, $\mathrm{r}=-0.14, \mathrm{p}=0.27$ )." should be read as "We found a significant correlation between the grooming and proximity distance networks (QAP test, $r$ = $0.51, \mathrm{p}<0.01)$ but not between the grooming and interindividual networks (QAP test, $r=-0.14, p=0.27$ ). [bold text used to highlight problem area]

Publisher's note Springer Nature remains neutral with regard to jurisdictional claims in published maps and institutional affiliations.

The online version of the original article can be found at https://doi.org/ 10.1007/s13364-018-0400-2

Sota Inoue

sota.inoe@gmail.com

1 Wildlife Research Center, Kyoto University, 2-24 Tanaka

Sekiden-cho, Sakyo, Kyoto 606-8203, Japan

2 Kyoto University Institute for Advanced Study, Yoshida Ushinomiya-cho, Sakyo, Kyoto 606-8501, Japan

3 Primates Research Institute, Kyoto University, 41-2 Kanrin, Inuyama 484-8506, Japan

4 Université Paris III Sorbonne Nouvelle, 13 rue Santeuil, 75005 Paris, France 